\title{
Informalidad laboral en los departamentos de Norte de Santander, Nariño, La Guajira y Cesar*
}

\author{
Labor informality in Norte de Santander, \\ Nariño, La Guajira and Cesar - Colombia
}

\section{Informalidade do trabalho nos departamentos do Norte de Santander, Nariño, La Guajira e Cesar - Colombia}

Jorge Raúl Ramírez Zambrano** Mario de Jesús Zambrano Miranda*** Johanna Milena Mogrovejo**** Jorge Luis Carreño Montaño*****

Fecha de recepción: 18 de enero de 2016

Fecha de aceptación: 31 de mayo de 2016

\footnotetext{
* Investigación realizada en el marco de los proyectos: "Determinantes regionales de la informalidad laboral en los departamentos fronterizos colombianos" y "Economía sumergida e informalidad laboral en los departamentos de la región de la frontera colombovenezolana 2000-2014". Los autores agradecen al Centro de Investigaciones de la Universidad Libre (Seccional Cúcuta) y la VIcerrectoría de Investigaciones de la Univesidad Francisco de Paula Santander por su apoyo.

** Docente e investigador de la Universidad Francisco de Paula Santander. Cúcuta - Colombia. Correo electrónico, jramirez7801@ gmail.com

*** Docente e investigador de la Universidad Libre Seccional Cúcuta -Colombia. Correo electrónico, mario.zambrano@unilibrecucuta. edu.co

**** Docente e investigadora de la Universidad Francisco de Paula Santander. Cúcuta Colombia. Correo electrónico, johannamogrovejo@ufps.edu.co

***** Estudiante de Economía de la Universidad de Pamplona y joven investigador. Correo electrónico, carrenojorge7@gmail.com
} 


\section{Resumen}

El artículo tiene como objetivo analizar la informalidad laboral desde un contexto regional, que abarca los departamentos de las zonas de frontera, mediante una metodología de tipo no paramétrico y otra de corte paramétrico. Dentro de la metodología no paramétrica se usa la elasticidad arco y en la paramétrica se utiliza el método de datos de panel. Los resultados muestran una relación positiva entre la participación del PIB industrial, el coeficiente de Gini, la pobreza monetaria y la informalidad laboral para los departamentos objeto de análisis.

Palabras clave: informalidad, seguridad social, teoría institucional, teoría estructuralista, economía regional.

JEL: C25, J01, J21, J31, J42.

\section{Abstract}

The article aims to analyze labor informality from a regional context including the states of border areas, through two kinds of methodology: non-parametric and parametric. Within the non-parametric methodology it is used the arc elasticity and in the parametric it is used panel data method. The results show a positive relationship between the participation of industrial GDP, the Gini coefficient, the monetary poverty and informal work for the states under review.

Keywords: informality, social security, institutional theory, structuralist theory, regional economy. 


\section{Resumo}

$\mathrm{O}$ artigo tem por objetivo analisar a informalidade do trabalho a partir de um contexto regional, que inclui os departamentos das áreas de fronteira, mediante uma metodologia de tipo paramétrica e outra de corte paramétrico. Dentro da metodologia não-paramétrica se usa a elasticidade arco e na paramétrica utiliza-se o método de dados de painel. Os resultados mostram uma relação positiva entre a parcela do PIB industrial, o coeficiente de Gini, a pobreza monetária e do trabalho informal para os departamentos em análise.

Palavras-chave: informalidade, segurança social, informal, teoria institucional, teoria estruturalista, economia regional. 


\section{INTRODUCCIÓN}

La informalidad laboral ${ }^{1}$ es un fenómeno que ha suscitado un debate polémico, rico y fecundo. Diversas corrientes de pensamiento han abordado el fenómeno presente en los mercados laborales, especialmente de las economías en vía de desarrollo. Las principales perspectivas que han tratado de definir, medir y explicar el fenómeno de la informalidad laboral se pueden agrupar en dos: la institucionalista y la estructuralista.

El objetivo de la investigación es articular estas corrientes de pensamiento siguiendo a García (2005; 2008 y 2009) y Galvis (2012), para explicar los determinantes regionales de la informalidad laboral en los departamentos fronterizos de $\mathrm{La}$ Guajira, Cesar, Norte de Santander y Nariño durante el periodo 2009-2013. El documento se basa en la hipótesis de que la informalidad laboral es un fenómeno local, persistente, alto y heterogéneo; esta alude a que debe tenerse en cuenta las características sociales, económicas y geográficas de las poblaciones objeto de estudio.

Los datos utilizados son proporcionados por el Departamento Administrativo Nacional de Estadística (Dane), se emplean los datos de la Gran Encuesta Integrada de Hogares (GEIH) y de cuentas departa- mentales. Como proxy de la informalidad laboral departamental se toma la de cada capital y área metropolitana respectiva. Los resultados muestran que en los departamentos de referencia se dan fallos estructurales y fallos institucionales de orden mixto (bajo desarrollo industrial y pobreza monetaria, y coeficiente de Gini), que deterioran las condiciones de vida de los habitantes de la frontera por la vía de la precarización, inestabilidad y carencia de ingresos, lo cual se evidencia en los altos y persistentes niveles de informalidad laboral.

La estructura del trabajo es la siguiente: en primer lugar se presenta esta introducción; luego se describe el contexto teórico con el que se aborda la informalidad laboral tanto de manera global como regional; enseguida se expone la metodología, la cual es de carácter paramétrico y no paramétrico; dentro de la metodología no paramétrica se realizan estimaciones a partir de la elasticidad arco, con el fin de encontrar ciertos comportamientos característicos e intrínsecos de cada unidad de análisis. Después se procede a las estimaciones de orden paramétrico, con la herramienta de datos de panel y el método de mínimos cuadrados agrupados; posteriormente se analizan los resultados. Por último, se concluye y se hacen algunas recomendaciones de política regional.

\footnotetext{
$1 \quad$ La definición usada en el desarrollo del trabajo será la expuesta por el DANE (2009), la cual define como trabajador informal: los trabajadores familiares sin remuneración, trabajadores por cuenta propia no profesionales ni técnicos, empleados domésticos, empleados y patrones de empresas del sector privado de menos de diez trabajadores.
} 


\section{MARCO TEÓRICO Y REVISIÓN LITERARIA}

\section{Marco teórico}

El origen del concepto de informalidad, según varios autores, surge en 1972 bajo una misión de empleo de la Organización Internacional del Trabajo (OIT) en Kenia, donde la OIT definió la informalidad como toda actividad que evade impuestos y no se somete a la regulación estatal. En paralelo a este estudio, Hart (1973) desarrolla una investigación en Ghana, donde analiza los negocios de pequeña escala y descubre que una porción considerable de la fuerza laboral urbana estaba trabajando por cuenta propia y fuera del mercado formal.

Este primer paso en la concepción de la informalidad laboral se aceptó en el mundo académico y progresivamente se empezó a despertar el interés por el tema. En este proceso de conceptualización teórica se han configurado dos corrientes de pensamiento: la estructuralista y la institucional. Ambas definen y explican desde su punto de vista las causas y los determinantes de la informalidad laboral.

La perspectiva estructuralista alude que las causas de la informalidad laboral radican en la insuficiencia estructural de las economías, la cual se desarrolla bajo dos enfoques: el enfoque de la demanda y el de oferta laboral. El enfoque de la demanda laboral asume que la estructura económica es incapaz de absorber la fuerza laboral disponible, lo que impulsa a la población restante a laborar en actividades precarias con el ánimo de subsistir o como una forma de evadir el desempleo (Perry et al., 2007). En este sentido, la perspectiva de la oferta laboral argumenta que la fuerza de trabajo no posee el capital humano requerido por el aparato productivo, y es por ello que la fuerza laboral no puede vincularse en empleos formales.

Por otro lado, la perspectiva institucional plantea la existencia de una relación positiva entre la informalidad laboral y la forma como los agentes eligen bajo una racionalidad ligada al análisis de costobeneficio $^{2}$. Esta perspectiva arguye que el fenómeno de la informalidad laboral se da bajo una decisión voluntaria de los individuos, basándose en los beneficios que la informalidad les ofrece, y los costos que les impone el Estado por la formalización. En esta concepción es el Estado el que genera las distorsiones del mercado con la imposición de normas y regulaciones que incrementan los costos para el empresariado y por ello recurren a la informalidad (Hirschman, 1970).

Ambas perspectivas poseen un punto de partida divergente a la hora de definir al trabajador informal, porque desde el enfoque institucional se concibe al individuo informal como emprendedor, informal por decisión, de ingresos mediosaltos y que presenta un comportamiento

Esto denota una noción y un factor determinante de la informalidad empresarial. 
procíclico frente a la evolución de la actividad económica. Por otro lado, los estructuralistas plantean que quienes recurren a la informalidad laboral, lo hacen por necesidad; bajo esta perspectiva los informales son individuos de ingresos bajos y su comportamiento es anticíclico al crecimiento económico.

Siguiendo a Galvis (2012) y Uribe y Ortiz (2006), las anteriores perspectivas explican la informalidad laboral desde diferentes puntos de vista. Sin embargo, en realidad ambos enfoques explican de manera conjunta el fenómeno de la informalidad laboral. Un punto de partida importante para interrelacionar los conceptos de cada enfoque es la heterogeneidad con que se ha calificado la informalidad laboral. La heterogeneidad de la informalidad laboral expone que las distorsiones del mercado laboral son creadas por las características socioeconómicas y geográficas propias de las regiones (efectos locales), como por ejemplo, cercanía a otro centro poblado, flujos migratorios (Galvis, 2002), ser un área limítrofe, la estructura productiva y tasa de cambio, García $(2005,2008$, 2009), Galvis (2012), Mora (2008), o por las rigideces provocadas dentro del mercado de trabajo a través de la excesiva intervención del Estado, lo cual ha sido planteado por Mondragón-Vélez y Peña (2010), Núñez (2002) y Flórez (2002).

\section{Revisión literaria}

El conjunto de artículos y documentos sobre la informalidad laboral en América
Latina y Colombia es extenso y profundo, para el caso de este trabajo solo se abordan los estudios que se tendrán como base del análisis. Los estudios fluctúan en su composición teórica entre el enfoque institucional, el estructural y mixto, y exponen metodologías y resultados consistentes con cada perspectiva.

Desde la perspectiva institucional, el Banco Mundial financió un estudio adelantado por Ribe et al. (2010), quienes argumentan que la informalidad laboral puede ser subsanada ampliando la cobertura de protección y eliminado las brechas de acceso y de prestación que inducen a la inequidad. En Colombia se han desarrollado diversas investigaciones que abordan la informalidad laboral desde hipótesis que aluden a la eficiencia de los mercados, la racionalidad de los agentes, la tasa de impuesto óptima, los excesos de intervención estatal, el incumplimiento de normas de contratación y de seguridad social. En esta línea se encuentran Núñez (2002), Guevara (2003), Uribe, Ortiz y Correa (2004), Farrell (2004), Amarante, Arim y Santamaría (2005), Mejía y Posada (2007), Perry et al. (2007), Cárdenas y Mejía (2007), Cárdenas y Rozo (2009), Bernal (2009), Sánchez, Duque y Ruiz (2009), Mondragón-Vélez y Peña (2010), Del Valle (2010) y Loayza y Rigolini (2011).

Desde la perspectiva estructuralista, Beccaria y Groisman (2008) y Gasparini y Tornarolli (2009) han desarrollado investigaciones para la economía latinoamericana, y han encontrado que el fenómeno es persistente y se halla aso- 
ciado a las estructura de la economía y al mercado de trabajo. En Colombia están las investigaciones de Ochoa y Ordóñez (2004), García (2005), Gómez (2007), Ortiz, Uribe y García (2007), Ortiz, Uribe y Badillo 2009), Uribe, Ortiz y García (2008), Vásquez y Ospino (2009), Figueroa (2010), y Aguilar, Hernández y Ramírez (2010).

Dada la heterogeneidad y persistencia de la informalidad laboral en Colombia, se han diseñado enfoques mixtos que combinan la perspectiva institucional y estructuralista, como son los casos de Flórez (2002), Uribe y Ortiz (2006), García (2008, 2009), Roldán y Ospino (2009), Bustamante (2011), Guataquí, García y Rodríguez (2011), Jiménez Restrepo (2011), Galvis (2012) y Quejada et al. (2014).

Es importante destacar que este artículo sigue en el nivel individual las dos perspectivas y asume una visión mixta. Al respecto, Flórez (2002) estudia la evolución de los componentes internos del empleo informal, y para ello divide en tres grupos la informalidad: empleos de subsistencia, asalariados informales o subordinados y pequeños empresarios. Flórez (2002) categorizó los informales de acuerdo con los datos provenientes de Encuesta Nacional de Hogares (ENH) de las diez principales ciudades para el periodo de 1984-2000. La investigadora concluye que la magnitud del sector informal para el 2000 es semejante al de
$1984^{3}$, y señala que los grupos de empleo de subsistencia son contracíclicos y el resto de grupos son procíclicos.

Otro aporte importante lo realizó García (2005) utilizando los datos de la ENH para las diez principales áreas metropolitanas en el periodo 1988-2000. El autor examina la relación entre la informalidad laboral urbana y el porcentaje de participación del PIB industrial a nivel departamental. Los resultados del autor demuestran una relación inversa entre la participación del PIB industrial y la informalidad laboral. Hay que aclarar que el autor toma la participación del PIB industrial como indicador del desarrollo de la estructura productiva de cada región. También demuestra que algunas características de las regiones pueden incidir en la informalidad laboral.

Dentro del mismo contexto, Uribe y Ortiz (2006) definen como principal característica de los trabajadores informales, la heterogeneidad. Los autores se basan en el mismo argumento expuesto por Flórez (2002), en donde una porción de la informalidad laboral presenta una tendencia procíclica y otra es contracíclica, dichos autores también indican que existen diversos determinantes que afectan de manera distinta a los trabajadores del sector informal.

Posteriormente, utilizando una perspectiva mixta, García (2009) incluyó variables a nivel agregado, siendo este trabajo una

\footnotetext{
Esto evidencia la persistencia de la informalidad laboral en Colombia.
} 
extensión de sus anteriores estudios (2005 y 2008). El autor utiliza datos del Dane para el periodo 1988-2006. García (2009) descubrió: “...que una mayor dinámica industrial, mayores esfuerzos del gobierno para evitar la informalidad, mayor presencia institucional, sin sobrepasar los límites del tamaño del sector público, y mayor dotación de capital humano de la población ocupada, frenan el crecimiento de las actividades informales" (p. 21). De igual manera, resalta que existen factores regionales que inciden en la informalidad laboral, y que estos pueden estar asociados a la especialización en actividades comerciales, la composición sectorial de la producción y la ubicación geográfica de cada área de análisis.

Galvis (2012, p. 6) considera que "en la realidad los enfoques se cruzan; por ejemplo, los institucionalista vinculan la informalidad con aspectos jurídicos y legales, pero tiene que enfrentar la pobreza y la necesidad de subsistencia, fundamento de los estructuralistas".

\section{METODOLOGÍA}

La metodología utilizada en este trabajo está dividida en dos partes: la primera es de orden no paramétrico y la segunda es de orden paramétrico. La metodología no paramétrica emplea la estimación de elasticidades mediante el método arco, mientras que la metodología paramétrica usa la herramienta de datos de panel a través del método de mínimos cuadrados agrupados.

\section{Elasticidad arco}

La elasticidad arco se utiliza para estimar las variaciones de la variable endógena sobre la variable explicativa; después se multiplica por el promedio inverso del cociente anterior. La descripción matemática es la siguiente:

$$
E l=\frac{\Delta Y i}{\Delta \text { variable Xi. }} * \frac{\text { promedio variable } X i}{\text { promedio } Y i}
$$

La elasticidad arco es calculada a lo largo de un intervalo de valores, en lugar de elegir el valor inicial o final se utiliza una medida de los dos, que es el promedio de cada variable. Las variables utilizadas en la estimación que simulan las Xi son la pobreza monetaria, el coeficiente de Gini y la participación del PIB industrial para cada departamento i, mientras que la Yi es la informalidad laboral de cada capital ciudad-área que se asume como proxy $\mathrm{de}^{4}$ cada departamento i.

\footnotetext{
$\overline{4} \quad$ Esta idea se basa en que las áreas metropolitanas concentran el mayor porcentaje de la población de cada departamento, a su vez, las actividades económicas de aglomeración se desarrollan en estos centros urbanos. Por otro lado, los flujos migratorios intradepartamentales se caracterizan por presiones del campo a la ciudad. Un aspecto característico de los departamentos localizados en zonas de frontera es la alta movilidad del trabajador fronterizo, entiéndase como el individuo que labora en un país y reside en el otro, lo propio aplica para los municipios.
} 
Por este método se calcula una elasticidad poco menos sesgada, lo que mejora la eficiencia del indicador. Los datos corresponden al periodo 2009-2013 y fueron suministrados por el Dane.

Al usar las elasticidades se busca medir la sensibilidad que experimenta la tasa de informalidad cuando varía el coeficiente de Gini, la participación del PIB industrial y la pobreza monetaria en los departamentos fronterizos. Esto nos permite mostrar provisionalmente indicios de que existen efectos locales y temporales para cada una de las unidades de análisis.

\section{Metodología de datos de panel}

El método utilizado es el de mínimos cuadrados agrupados, el cual es un método básico para estimar un modelo de datos de panel, que "incluye una muestra de agentes económicos o de interés (individuos, empresas, bancos, ciudades, países, etc.) para un período determinado de tiempo, esto es, combina ambos tipos de datos (dimensión temporal y estructural)" (Mayorga \& Muñoz, 2000, p. 1).

El objeto del modelo de datos de panel "es capturar la heterogeneidad no observable, ya sea entre agentes económicos o de estudio, así como también en el tiempo, dado que esta heterogeneidad no se puede detectar ni con estudios de series temporales ni tampoco con los de corte transversal..." (Mayorga \& Muñoz, 2000, p. 2).

Según Mayorga y Muñoz (2000), el modelo de datos de panel permite realizar: ...un análisis más dinámico al incorporar la dimensión temporal de los datos, lo que enriquece el estudio, particularmente en períodos de grandes cambios. Esta modalidad de analizar la información en un modelo de panel es muy usual en estudios de naturaleza microeconómica. La aplicación de esta metodología permite analizar dos aspectos de suma importancia cuando se trabaja con este tipo de información y que forman parte de la heterogeneidad no observable: i) los efectos individuales específicos y ii) los efectos temporales. En lo que se refiere a los efectos individuales especificos, se dice que estos son aquellos que afectan de manera desigual a cada uno de los agentes de estudio contenidos en la muestra (individuos, empresas, bancos) los cuales son invariables en el tiempo y que afectan de manera directa las decisiones que tomen dichas unidades. Usualmente se identifica este tipo de efectos con cuestiones de capacidad empresarial, eficiencia operativa, capitalización de la experiencia, acceso a la tecnología, etc. Los efectos temporales serían aquellos que afectan por igual a todas las unidades individuales del estudio pero que no varían en el tiempo. Este tipo de efectos pueden asociarse, por ejemplo, a los choques macroeconómicos que pueden afectar por igual a todas las empresas o unidades de estudio. (2000, p.2) 
De manera que la técnica de datos de panel permite evaluar los efectos tanto fijos como temporales que están asociados a la pobreza monetaria, la participación del PIB y el coeficiente de Gini, y esta técnica, a su vez, conduce a valorar dichos efectos en la informalidad laboral, dado que los departamentos colombianos localizados en zona de frontera presentan características heterogéneas, específicas y diferenciadas que son inherentes a la dinámica de la economía informal.

Un modelo de datos de panel puede expresarse de forma genérica de la siguiente manera:

$$
\mathrm{Y}_{\mathrm{it}}=\alpha_{\mathrm{it}}+\mathrm{X}_{\mathrm{it}} \beta++\mathrm{U}_{\mathrm{it}}(1)
$$

Con $i=1, \ldots N ; t=1, \ldots T$.

\section{Donde:}

i: se refiere al departamento fronterizo,

t: a la dimensión en el tiempo,

$\alpha$ : es un vector de interceptos de $n$ parámetros,

b: es un vector de $\mathrm{K}$ parámetros $\mathrm{y}$,

$\mathrm{Y}_{\mathrm{ti}}$ es el índice de informalidad laboral para Norte de Santander, Nariño, La Guajira y Cesar durante el periodo 2009 a 2013 según metodología adoptada por el Dane (2009).

$\mathrm{X}_{\mathrm{it}}$ : es la i-ésima observación al momento t para las $\mathrm{K}$ variables explicativas; compuesta por pobreza monetaria, el coeficiente de Gini, la participación del sector industrial en el producto interno bruto de cada departamento $i$.

Para este caso, la muestra total de las observaciones en el modelo vendría dado por $\mathrm{N}$ x T. El término de error $\mathrm{U}_{\mathrm{it}}$ incluido en la ecuación (1), puede descomponerse de la siguiente manera:

$$
\mathrm{U}_{\mathrm{it}=} \mu_{\mathrm{i}}+\delta_{\mathrm{t}}+\mathcal{E}_{\mathrm{it}}(2)
$$

$\mu_{\mathrm{it}}$ : representa los efectos no observables que difieren entre las unidades de estudio pero no en el tiempo, que generalmente se los asocia a cada departamento.

$\delta_{\text {t: }}$ se le identifica con efectos no cuantificables que varían en el tiempo pero no entre los departamentos fronterizos, objeto de estudio.

$\boldsymbol{\varepsilon}_{\mathrm{it}}$ : se refiere al término de error específicamente aleatorio.

El modelo se plantea bajo las siguientes consideraciones teóricas: bajo la perspectiva estructural, cuando se desarrollan actividades industriales formales en los departamentos objeto de estudio, el efecto sobre la tasa de informalidad será negativo. Ahora bien, en la medida que haya una modesta y débil especialización industrial, dicho efecto será positivo, debido a que la mera presencia del PIB industrial no garantiza que la generación de empleos sea formal, por tanto su efecto será menor y positivo sobre la tasa de informalidad laboral, habida cuenta de que en los departamentos estudiados, el departamento "de mejor" desempeño industrial es Norte de Santander y este se encuentra rezagado frente a los departamentos colombianos líderes en la materia. Por otro lado, el rasgo distintivo de las actividades industriales en los departamentos fronterizos, es su baja complejidad, baja generación de valor y su escasa creación de empleos tanto en cantidad como en calidad. En este 
aspecto, el trabajo se aleja de la perspectiva planteada por García $(2005,2008$ y 2009), aunque se mantiene la hipótesis de corte estructuralista.

Bajo este mismo enfoque y siguiendo a Galvis (2012), la variable pobreza se asume como proxy de las carencias de ingresos y la necesidad de subsistencia que conduce al individuo a engancharse en actividades económicas precarias y de baja productividad, es decir, informales. A su vez, un rasgo característico de la población pobre es su escasa escolaridad. En este escenario, los individuos "eligen" las actividades informales bajo un esquema de racionalidad acotada y condicionada ${ }^{5}$ para generar ingresos.

La variable coeficiente Gini se utiliza como proxy de la capacidad estatal y del mercado, para resolver los desequilibrios sociales producidos por la segmentación, la precarización de las condiciones laborales y de ingresos a una escala regional, lo cual se analiza desde una perspectiva mixta. El efecto conjunto esperado es un círculo vicioso donde persiste la informalidad laboral, la pobreza y la desigualdad de ingresos, lo que amplía las brechas y asimetrías regionales del país.

\section{Datos y estadística descriptiva}

Los datos utilizados provienen del Dane a través de la GEIH y las cuentas departamentales, el periodo de análisis contempla los años 2009-2013. La estimación de la media y la desviación estándar se realiza con el fin de caracterizar las variables exógenas usadas en el análisis. Los datos correspondientes a la variable informalidad laboral no están para el agregado departamental, por tanto se toman los datos de cada ciudad-área como variable proxy.

Tabla 1. Estadística descriptiva del periodo 2009-2013

\begin{tabular}{|l|c|c|c|c|c|c|c|c|}
\hline \multirow{2}{*}{ Departamento } & \multicolumn{2}{|c|}{$\begin{array}{c}\text { Tasa de informa- } \\
\text { lidad laboral }\end{array}$} & \multicolumn{2}{c|}{$\begin{array}{c}\text { Coeficiente } \\
\text { de Gini }\end{array}$} & \multicolumn{2}{c|}{$\begin{array}{c}\text { Pobreza } \\
\text { monetaria }\end{array}$} & \multicolumn{2}{c|}{$\begin{array}{c}\text { Participación } \\
\text { PIB industrial }\end{array}$} \\
\cline { 2 - 9 } & Media & $\begin{array}{c}\text { Des- } \\
\text { viación } \\
\text { estándar }\end{array}$ & Media & $\begin{array}{c}\text { Des- } \\
\text { viación } \\
\text { estándar }\end{array}$ & Media & $\begin{array}{c}\text { Des- } \\
\text { viación } \\
\text { estándar }\end{array}$ & Media & $\begin{array}{c}\text { Des- } \\
\text { viación } \\
\text { estándar }\end{array}$ \\
\hline $\begin{array}{l}\text { Norte de } \\
\text { Santander }\end{array}$ & 68,5 & 0,025 & 0,497 & 0,015 & 43,617 & 4,533 & 7,49 & 0,558 \\
Nariño & 63,1 & 0,019 & 0,509 & 0,011 & 52,767 & 3,606 & 5,17 & 0,406 \\
La Guajira & 66,4 & 0,014 & 0,577 & 0,0217 & 61,380 & 5,130 & 0,99 & 0,079 \\
Cesar & 65,9 & 0,016 & 0,522 & 0,024 & 52,367 & 7,385 & 3,58 & 0,293 \\
\hline
\end{tabular}

Fuente: cálculos propios con base en DANE.

\footnotetext{
$5 \quad$ Las decisiones se dan en un ambiente de incertidumbre e inseguridad generado por la carencia de ingresos. La informalidad laboral es la única oportunidad en medio de la pobreza y el desempleo.
} 
La Tabla1 muestra los valores descriptivos para cada variable, indica la persistencia de la informalidad laboral en los departamentos objeto de estudio. Al respecto, la tasa de informalidad laboral (promedio) más alta se presenta en Norte de Santander $(68.5 \%)$, con una desviación estándar de 0.025 . La media de la tasa de informalidad más baja se manifiesta en el departamento de Nariño, con un valor de $63.1 \%$, su desviación es de 0.019. Los valores medios de los cuatro departamentos durante el periodo de análisis son superiores al $60 \%$ con respecto al total de ocupados según cada caso.

Además, la Tabla 1 revela los promedios de los coeficientes de Gini para los cuatro departamentos fronterizos; la media más baja la obtiene Norte de Santander (0.497) y el mayor valor se presenta en el departamento de La Guajira con 0.577.

Los resultados de las estadísticas descriptivas revelan que para el caso de la pobreza monetaria, la media más baja se presenta nuevamente en Norte de Santander con un valor de $43.6 \%$. Sin embargo, el valor más alto lo obtiene La Guajira (61.38 \%),

En otras palabras, la pobreza monetaria que se presenta en los cuatro departamentos es muy alta, lo que evidencia que aproximadamente más de la mitad de la población colombiana que habita en zona de fronteriza es pobre a causa de la insuficiencia de ingresos.

Bajo esta línea de análisis y teniendo en cuenta la variable PIB industrial, se observa que el mayor grado de desarrollo manufacturero lo alcanza Norte de Santander con un valor medio de $7.49 \%$ con respecto a su PIB. Mientras que el valor promedio más bajo se presenta en La Guajira (0.99 \%).

En síntesis, puede argumentarse que el departamento que presenta "mejores" indicadores es Norte de Santander. Sin embargo, Norte de Santander es el departamento con mayores índices de informalidad laboral. Por otro lado, el departamento de La Guajira muestra los peores indicadores en pobreza, desigualdad y desarrollo industrial y, a su vez, posee la segunda tasa de informalidad más alta.

\section{RESULTADOS}

El cálculo de las elasticidades muestra el impacto de cada coeficiente en las variables independientes sobre la informalidad laboral, por lo cual se estiman los índices para cada departamento fronterizo. En la Tabla 2 se pueden apreciar los coeficientes de impacto sobre la informalidad laboral en relación con la distribución del ingreso. En primer lugar, se encuentra que los coeficientes presentan un impacto negativo, algo que no se esperaría debido a la relación esperada según el planteamiento teórico que asume este trabajo. La Tabla 2 también muestra la existencia de coeficientes con signos positivos, sin embargo, es mayor la cantidad de elasticidades negativas; para el caso de Norte de Santander, los valores correspondientes a los años 2010, 2012 y 2013 presentan un signo negativo. 
Con base en lo anterior, se puede afirmar para los años referenciados que un crecimiento de la desigualdad en $1 \%$, genera una reducción en la informalidad laboral de $-0,24 \%,-0.937 \%$ y $-0,73 \%$. Caso contrario ocurriría en el resto de años.

La elasticidad arco estimada para Nariño muestra que la totalidad de los coeficientes calculados presentan signo negativo, teniendo como su máximo valor un coefi- ciente de -11.381 en 2012, lo que quiere decir, que un aumento de la desigualdad del ingreso de $1 \%$ está asociado a una reducción en la informalidad laboral de $11.38 \%$.

Los valores calculados para La Guajira y Cesar son comparativamente similares, en su mayoría presentan valores positivos y negativos; otra regularidad es que los coeficientes no indican valores muy altos.

Tabla 2. Elasticidad informalidad laboral-coeficiente de Gini.

\begin{tabular}{|l|c|c|c|c|c|}
\hline $\begin{array}{c}\text { Elasticidad informalidad } \\
\text { laboral-coeficiente de Gini }\end{array}$ & $\mathbf{2 0 0 9}$ & $\mathbf{2 0 1 0}$ & $\mathbf{2 0 1 1}$ & $\mathbf{2 0 1 2}$ & $\mathbf{2 0 1 3}$ \\
\hline Norte de Santander & 0,081 & $-0,244$ & 3,261 & $-0,937$ & $-0,732$ \\
Nariño & $-2,306$ & $-0,735$ & $-1,549$ & $-11,381$ & $-1,134$ \\
La Guajira & $-0,104$ & 0,038 & 0,117 & 1,229 & $-2,098$ \\
Cesar & 0,324 & $-0,227$ & $-1,128$ & 0,931 & 1,031 \\
\hline
\end{tabular}

Fuente: cálculos propios con base en DANE.

Tabla 3. Elasticidad informalidad laboral-participación PIB industrial.

\begin{tabular}{|l|c|c|c|c|c|}
\hline $\begin{array}{c}\text { Elasticidad informalidad } \\
\text { laboral-participación PIB } \\
\text { industrial }\end{array}$ & $\mathbf{2 0 0 9}$ & $\mathbf{2 0 1 0}$ & $\mathbf{2 0 1 1}$ & $\mathbf{2 0 1 2}$ & $\mathbf{2 0 1 3}$ \\
\hline Norte de Santander & $-0,023$ & $-0,302$ & 2,300 & $-0,611$ & $-0,126$ \\
Nariño & $-0,477$ & $-0,589$ & 0,633 & 1,372 & 0,937 \\
Guajira & 0,023 & $-0,070$ & 0,065 & $-0,419$ & $-0,223$ \\
Cesar & 0,919 & $-0,103$ & 0,102 & 0,491 & $-0,429$ \\
\hline
\end{tabular}

Fuente: cálculos propios con base en DANE.

La Tabla 3 revela que los signos de los coeficientes son heterogéneos para cada departamento fronterizo. Al respecto, Norte de Santander presenta tres valores negativos para los años 2009, 2010 y 2013 , lo cual indica una relación inversa entre la participación del PIB industrial y la informalidad laboral. Este comportamiento ha sido estudiado de manera continua mediante la hipótesis según la cual el sector industrial refleja el sector moderno de la economía. 
En los datos estimados para Norte de Santander se observan solamente para los valores negativos unos coeficientes de impacto menores a 0,5 , en otras palabras, el PIB industrial está asociado marginalmente a la informalidad laboral.

De igual manera, Norte de Santander presenta valores positivos en las elasticidades de los años 2011 y 2012 respectivamente. De forma muy singular, en el año 2011, el índice de impacto fue de 2.3, lo cual denota una sensibilidad considerable en el deterioro y alza de la informalidad laboral.

Siguiendo con las estimaciones, el departamento de Nariño muestra dos valores negativos para los años 2009 y 2010, seguidamente los coeficientes cambian de signos y terminan la serie con valores positivos, para Nariño los coeficientes de impacto con signo negativo arrojan valores inferiores a 1 , mientras que los coeficientes positivos presentan un coeficiente por encima de 1. En este sentido, Nariño posee en los primeros años del análisis similitudes con los coeficientes estimados para Norte de Santander.

Para el caso de La Guajira y Cesar son semejantes las estimaciones, los coeficientes presentan valores inferiores a 1 y toman ambos signos. Las diferencias estriban en que el departamento de La Guajira presenta más coeficientes negativos que Cesar, y, a su vez estos departamentos presentan tasas similares de informalidad laboral.

Tabla 4. Elasticidad informalidad-pobreza monetaria.

\begin{tabular}{|l|c|c|c|c|c|}
\hline $\begin{array}{c}\text { Elasticidad informalidad } \\
\text { laboral-pobreza monetaria }\end{array}$ & $\mathbf{2 0 0 9}$ & $\mathbf{2 0 1 0}$ & $\mathbf{2 0 1 1}$ & $\mathbf{2 0 1 2}$ & $\mathbf{2 0 1 3}$ \\
\hline Norte de Santander & $-0,048$ & $-0,144$ & $-0,551$ & $-5,016$ & $-0,303$ \\
Nariño & $-0,683$ & $-0,255$ & 0,260 & 4,596 & 1,594 \\
Guajira & $-0,088$ & $-0,088$ & 0,077 & $-1,394$ & 0,494 \\
Cesar & $-0,270$ & $-0,037$ & 0,096 & 2,789 & 0,663 \\
\hline
\end{tabular}

Fuente: cálculos propios con base en DANE.

En la Tabla 4 se exhiben las estimaciones de los índices de impacto con respecto a la informalidad laboral, tomando como variable explicativa la pobreza monetaria. En primer lugar, se observa que para Norte de Santander todos los datos estimados son negativos, de igual manera la mayoría de sus valores son inferiores a
1 , con excepción del coeficiente estimado para 2012, que presenta un valor de -5 puntos. Un efecto local que se evidencia en Norte de Santander es la relación negativa entre la informalidad y la pobreza monetaria, algo que va en contra de los planteamientos teóricos de esta investigación. 
En cambio, Nariño no muestra la totalidad de sus coeficientes con signo negativo, incluso son mayores los coeficientes positivos en los años analizados. Nariño arroja coeficientes positivos para los años 2011, 2012 y 2013, los cuales son superiores a 1 , mientras que los coeficientes negativos son inferiores a 1 .

Los departamentos de La Guajira y Cesar presentan en su mayoría coeficientes inferiores a 1 , solamente para el año 2012 se da un valor mayor a 1 , pero los valores divergen en el signo. Al igual que en las estimaciones anteriores estos departamentos también presentan similitudes en el cálculo de las elasticidades.

Otra regularidad se observa para los años 2009 y 2010, los datos calculados para los cuatro departamentos fronterizos presentan signo negativo. En síntesis, las estimaciones no paramétricas muestran ciertos efectos o comportamientos heterogéneos en la informalidad laboral en relación con las variables exógenas, pero estos efectos se dan de manera diferenciada y quizás localizada, tanto por departamento como por año.

Al presentarse estos efectos asimétricos y heterogéneos, se da una colisión preliminar con las hipótesis planteadas, pero las regularidades observadas en cada departamento ofrecen al mismo tiempo una ventana de oportunidad para generar un análisis más riguroso y aproximativo que permita una mayor comprensión del fenómeno de la informalidad laboral en los departamentos colombianos localizados en zonas de frontera. Por lo anterior, se recurre a la metodología de corte paramétrico a través del modelo de datos de panel.

El modelo general de datos de panel estimado calcula la incidencia de las tres variables independientes (pobreza monetaria, participación del PIB industrial y coeficiente de Gini) sobre la informalidad laboral para los departamentos de Nariño, Cesar, La Guajira y Norte de Santander.

En la Tabla 5 se observa la estimación resultante aplicando la metodología de datos de panel; lo primero que se puede extraer es la significancia individual que tiene cada variable dentro del modelo, dado que los t-estadísticos son considerablemente buenos, lo cual revela el grado de asociación con la informalidad laboral en los departamentos fronterizos. Del mismo modo se puede notar que el coeficiente de determinación del modelo arroja un valor de 0,3297-, un coeficiente de relevancia conjunta aceptable dado el modelo adoptado en la investigación, lo cual da cabida a mejoras estadísticas y econométricas a partir de la ampliación del número de observaciones y de la calidad de las variables con el fin de incrementar el ajuste del modelo. 
Informalidad laboral en los departamentos de Norte de Santander, Nariño, La Guajira y Cesar

Jorge Ramírez - Mario Zambrano - Johanna Mogrovejo - Jorge Carreño

Tabla 5. Regresión general a través del modelo datos de panel.

\begin{tabular}{|c|c|c|c|}
\hline Variable & Coeficiente & t-estadístico & Probabilidad \\
\hline Pobreza & 0.004458 & 2.095550 & 0.0524 \\
\hline Gini & 0.626713 & 3.001497 & 0.0085 \\
\hline PIB industrial & 0.034476 & 3.867306 & 0.0014 \\
\hline AR(1) & 0.694632 & 6.528669 & 0.0000 \\
\hline Variable dependiente & Informalidad laboral & R2 & 0.329 \\
\hline Efectos fijo por departamento & \multicolumn{2}{|c|}{ coeficiente } \\
\hline Norte de Santander & \multicolumn{2}{|c|}{0.017} \\
Nariño & \multicolumn{2}{c|}{0.0094} \\
Cesar & \multicolumn{2}{c}{0.037} \\
La Guajira & \multicolumn{2}{c}{} \\
\hline
\end{tabular}

Fuente: cálculos propios a partir de las regresiones.

\section{DISCUSIÓN}

Las estimaciones econométricas evidencian que las variables: grado de especialización de las actividades industriales a razón del PIB departamental, las variables Gini y pobreza monetaria generan un efecto positivo sobre la tasa de informalidad laboral en cada uno de los departamentos fronterizos. Es decir, que ante un aumento de $1 \%$ en las actividades industriales, del coeficiente de Gini (baja capacidad estatal y privada), de la pobreza (vía de escape), se genera un incremento de $0,034 \%, 0,620,004 \%$ en la informalidad laboral. Siendo mayor la incidencia del coeficiente de Gini y con ello la baja capacidad del Estado y del mercado a nivel local para resolver los desequilibrios sociales, laborales y de ingresos que caracterizan a los departamentos de Norte de Santander, Nariño, La Guajira y Cesar.
El primer hallazgo contradice los resultados surgidos de las estimaciones no paramétricas y los resultados planteados por García (2005, 2008 y 2009), lo cual es consistente con las hipótesis planteadas en este trabajo. El segundo y tercer hallazgo son interesantes en la medida en que la investigación logra demostrar que en los departamentos de referencia se dan fallos institucionales de orden mixto que deterioran las condiciones de vida de los habitantes de la frontera, por la vía de la precarización, la inestabilidad y los bajos ingresos, evidenciado en los altos y persistentes niveles de informalidad laboral.

El efecto conjunto de las variables y la calidad de los estimadores es aceptable teniendo en cuenta los valores alcanzados por el coeficiente de determinación ${ }^{6}$. Este resultado contrasta con los t-estadísticos de los parámetros individuales.

\footnotetext{
6 Debe destacarse que una de las limitaciones del trabajo es este resultado, debido a las dificultades encontradas al construir la serie de datos con información estadística más amplia y rica. Lo cual se convierte en una oportunidad para mejorar en futuros trabajos.
} 
Ahora bien, al aplicar el modelo de panel con efectos fijos sobre los departamentos, los estimadores pueden indicar que la heterogeneidad y especificidad de la informalidad laboral tiene su explicación no en la hipótesis que defiende aspectos atinentes a la localización geográfica, sino que la explicación está asociada al funcionamiento y calidad de las instituciones regionales para resolver los problemas que caracterizan a las estructuras económicas, sociales y políticas de los departamentos fronterizos colombianos.

\section{CONCLUSIONES}

Este trabajo contribuye al análisis de los determinantes regionales de la informalidad laboral en Colombia, con especial atención en los departamentos localizados en zonas de frontera donde se presentan los mayores índices de precarización e inestabilidad laboral del país. Los resultados no paramétricos revelan que existe un comportamiento heterogéneo, específico y asimétrico muy característico de las unidades de estudio.

Los resultados, usando la metodología paramétrica, evidencian que tanto la hipótesis institucional, estructuralista, o la seguida en este trabajo, bajo el enfoque mixto, son pertinentes y válidas. Es decir, que los fallos institucionales tanto del Estado como del mercado a nivel regional repercuten de manera significativa en los logros laborales de los departamentos fronterizos. De manera que una mayor presencia institucional en el sector público para responder a las demandas sociales, combinada con el florecimiento de una clase empresarial audaz, innovadora, transformadora y generadora de valor, puede incidir de forma positiva tanto en la especialización en actividades industriales complejas como en la generación de empleos estables y de calidad.

Los efectos fijos parecen tener influencia sobre la informalidad laboral regional, se considera que en adelante los trabajos sobre la materia deben tratar de ampliar la cantidad y calidad de los datos, así mismo, aumentar las unidades de análisis, habida cuenta de que la otra limitación del trabajo es no haber incluido la minería de datos correspondientes al resto de departamentos fronterizos, debido a las restricciones que presentan los datos provenientes del Dane a partir de la recolección de la información vía GEIH.

De manera general y según los resultados, se necesita una política industrial territorial, en especial en las zonas de frontera, para generar procesos de desarrollo y crecimiento económico, y posibilitar mejores condiciones de vida de los ciudadanos. Hacer frente a la informalidad laboral y sus consecuencias dadas las características territoriales de los departamentos analizados, es complejo y pasa por un análisis territorial, integral y dinámico, que exige la complementariedad del binomio Estado-mercado, esto es, una relación interna y sinérgica, que debe fundamentarse en el trípode Estadoempresa-academia. 
Las recomendaciones o los aspectos para tener en cuenta son los siguientes:

El Estado, en todos sus órdenes, debe tener una mayor presencia con una política social y económica de frontera, esto es, una política que busque reducir la informalidad laboral. Esto implica hacer una mayor inversión en educación tanto en cobertura como en calidad y por ende en pertinencia, acordes a las necesidades sociales y del aparato productivo en formación técnica y tecnológica, al igual que en formación universitaria de alto nivel. Con esto, mejora la cualificación de la mano de obra que exigen las actividades industriales complejas, que deben producir empleo de calidad para mitigar la informalidad laboral.

El desarrollo de economías de aglomeración, de economías de escala y externas son tareas pendientes de los hacedores de política regional y del sector privado, para ello deben definirse apuestas productivas con impacto local y territorial.

\section{REFERENCIAS}

Aguilar, J., Hernández, C. \& Ramírez, M. (2010). Efecto del ciclo económico en la incidencia de la informalidad: evidencia para México 1987-1999. Economía y Sociedad, 20.

Amarante, V., Arim, R. \& Santamaría, M. (2005). Los efectos de la reforma laboral de 2002 en el mercado laboral colombiano. (006). Perfil Económico, 8, 67-82.

Beccaria, L. \& Groisman, F. (2008). Informalidad y pobreza en Argentina. Investigación Económica, LXVII (266).

Bernal, R. (2009). The Informal Labor Market in Colombia: Identification and Characterization. Desarrollo y Sociedad, 63, 145-208.

Bustamante, J. (2011). Los retos de la economía informal en Colombia. Bogotá: Ministerio de Hacienda y Crédito Público, Dirección General de Política Macroeconómica.

Cárdenas, M. \& Mejía, C. (2007). Informalidad en Colombia: Nueva Evidencia. Documento de trabajo No. 35. Fedesarrollo-Banco Interamericano de Desarrollo, marzo.

Cárdenas, M. \& Rozo, S. (2009). Informalidad empresarial en Colombia: problemas y soluciones. Desarrollo y Sociedad 63 (primer semestre), 211-245. 
Dane (2009). Metodología informalidad laboral. Gran Encuesta Integrada de HogaresGEIH. Bogotá D.C.

Del Valle, A. (2010). Informalidad y modelos de bienestar en América Latina. Política y Sociedad, 23.

Farrell, D. (2004). The Hidden Dangers of the Informal Economy. McKinsey Quarterly.

Figueroa, C. (2010). Determinantes de la informalidad laboral y el subempleo en las áreas metropolitanas de Barranquilla, Cartagena y Montería. Documentos IEEC, Universidad del Norte.

Flórez, C. (2002). The Function of the Urban Informal Sector in Employment. Evidence from Colombia 1984-2000. Documentos CEDE, (4).

Galvis, Luis. A. (2002, junio). Determinantes de la migración interdepartamental en Colombia, 1988-1993. Revista de Economía del Rosario, 5(1), 93-118.

Galvis, L. A. (2012, feb.). Informalidad laboral en las áreas urbanas de Colombia. Documentos de Trabajo de Economía Regional, (164), 1-52.

García, G. (2005). El componente local de la informalidad laboral para las diez principales áreas metropolitanas de Colombia, 1988-2000. Desarrollo y Sociedad. Recuperado de http://redalyc.uaemex.mx/src/inicio/ArtPdfRed. jsp?iCve $=169114671004$

García, G. (2008). Informalidad regional en Colombia, evidencia y determinantes. Cali, Colombia: Centro de investigaciones y documentación socioeconómicaCIDSE, Universidad del Valle.

García, G. (2009). Evolución de la informalidad laboral en Colombia: determinantes macros y efectos locales. Archivos de Economía, DNP, (360).

Gasparini, L. \& Tornarolli, L. (2009). Labor informality in Latin America and the Caribbean: Patterns and Trends from Household Survey Microdata. Desarrollo y Sociedad, 69.

Gómez, L. G. (2007). La informalidad en la economía: algo incuestionable. Semestre Económico, 10(19).

Guataquí, J., García, A. \& Rodríguez, M. (2011). El perfil de la informalidad laboral en Colombia. Perfil de Coyuntura Económica 16, 91-115.

Guevara, D. A. (2003). Globalización y mercado de trabajo en Colombia: algunas consideraciones en el marco de la flexibilización laboral. Reflexión Política, 5(10). 
Hart, K. (1973). Informal Income Opportunities and Urban Employment in Ghana. The Journal of Modern African studies, 11(01), 61-89.

Hirschman, A. (1970). Exit, Voice, and Loyalty: Responses to decline in Firms, Organizations and States. Cambridge, MA: Harvard University Press.

Jiménez Restrepo, D. M. (2012). La informalidad laboral en América Latina: explicación estructuralista o institucionalista. Cuadernos de Economía, 31(58), 113-143.

Loayza, N. \& Rigolini, J. (2011). Informal Employment: Safety net or Growth Engine? World Development, 13.

Mayorga, M. \& Muñoz, E. (2000). La técnica de datos de panel: una guía para su uso e interpretación. Costa Rica: Banco de Central de Costa Rica, División Económica, Departamento de Investigaciones Económicas

Mejía, D. \& Posada, C. E. (2007). Informalidad: teoría e implicaciones de política. Borradores de Economía, 455, 1-33.

Mondragón-Vélez, C. \& Peña, X. (2010). Labor Market Rigidities and Informality in Colombia. The Journal of LACEA, Economía, 11(1).

Mora, A. F. (2008). Informalidad, política social y tipo de cambio: aportes para el análisis de la coyuntura colombiana actual. Revista Economía, Gestión y Desarrollo, (6), 357-386.

Núñez, J. (2002). Empleo informal y evasión fiscal en Colombia. Archivos de Economía DNP, (210).

Ochoa, D. \& Ordóñez, A. (2004). Informalidad en Colombia: causas, efectos y características de la economía del rebusque. Cali: Universidad ICESI.

Ortiz, C., Uribe, J. \& Badillo, É. (2009). Segmentación inter e intrarregional en el mercado laboral urbano de Colombia, 2001-2006. Ensayos sobre Politica Económica, 27(58), 194-231.

Ortiz, C., Uribe, J. \& García, G. (2007). Informalidad y subempleo: un modelo probit bivariado aplicado al Valle del Cauca. Sociedad y Economía, 13, 104-131.

Perry, G., Maloney, W., Arias, O. Fajnzylber, P., Mason, A. \& Saavedra-Chanduvi, J. (2007). Informalidad: escape y exclusión. Washington, D.C.: Banco Mundial. 
Ribe, H. Robalino, D. \& Walker, I. (2010). Hacia la protección social eficaz para todos en Amarica Latina y el Caribe. De los derechos a la realidad. Banco Mundial. Junio. Recuperado de: http://www2.congreso.gob.pe/sicr/cendocbib/con3_uibd. nsf/CB99A79DD574F8B2052578C000780103/\$FILE/De_los_Derechos_a la_Realidad[1].pdf

Roldán, P. \& Ospino, C. (2009). ¿Quiénes terminan en la informalidad?: impacto de las características y el tiempo de búsqueda. Revista de Economía del Caribe, 4, 149-180.

Quejada, R., Yánez, M. \& Cano, K. (2014). Determinantes de la informalidad laboral: un análisis para Colombia. Investigación \& Desarrollo, 22(1).

Sánchez, F., Duque, V. \& Ruiz, M. (2009). Costos laborales y no laborales y su impacto sobre el desempleo, la duración del desempleo y la informalidad en Colombia 1980-2007. Documento CEDE, 68.

Uribe, J., \& Ortiz, C. (2006). Informalidad laboral en Colombia, 1988-2000: evolución, teorías y modelos. Cali, Colombia: Universidad del Valle.

Uribe, J., Ortiz, C. \& Correa, J. (2004). Determinantes de las decisiones en el mercado laboral: la decisión de ser informal en Colombia 1988-2000. Documentos de Trabajo CIDSE, (79).

Uribe, J., Ortiz, C. \& García, G. (2008). Informalidad y subempleo: dos caras de la misma moneda. Cuadernos de Administración, 21(37), 211-241.

Vásquez, P. \& Ospino, C. (2009). ¿Quiénes terminan en la informalidad: impacto de las características y el tiempo de búsqueda. Revista de Economía del Caribe, 4. 